\title{
An Analytic-to-Holistic Approach for Face Recognition Based on a Single Frontal View
}

\author{
Kin-Man Lam, Member, IEEE, and Hong Yan, Senior Member, IEEE
}

\begin{abstract}
In this paper, we propose an analytic-to-holistic approach which can identify faces at different perspective variations. The database for the test consists of 40 frontal-view faces. The first step is to locate 15 feature points on a face. A head model is proposed, and the rotation of the face can be estimated using geometrical measurements. The positions of the feature points are adjusted so that their corresponding positions for the frontal view are approximated. These feature points are then compared with the feature points of the faces in a database using a similarity transform. In the second step, we set up windows for the eyes, nose, and mouth. These feature windows are compared with those in the database by correlation. Results show that this approach can achieve a similar level of performance from different viewing directions of a face. Under different perspective variations, the overall recognition rates are over 84 percent and 96 percent for the first and the first three likely matched faces, respectively.
\end{abstract}

Index Terms-Face recognition, facial feature detection, head model, point matching, correlation.

\section{INTRODUCTION}

$\mathrm{F}$ ACE recognition is an important capability of human beings in their social life. We can remember hundreds or even thousands of faces in our whole life and identify a face in different perspective variations, illuminations, ages, etc. Under very poor illumination conditions, a face can still be recognized, as the position of the different facial features and the face contours are usually sufficient for recognition. This suggests an approach to face recognition, whereby the geometrical positions of the different facial features are measured first, and then the details of each feature are used for further matching. This is also a common where-to-what approach for object recognition.

The problem of human face recognition [1] has been studied for more than 20 years. The existing approaches for face recognition may be classified into two categories, holistic and analytic. The holistic approaches consider the global properties of the pattern. Turk et al. [2], [3] proposed an eigenface system, which projects face images onto a feature space that spans the significant variations among known face images. Isodensity lines [4], which are the boundaries of constant gray level areas after quantizing an image, were also investigated for face recognition. Dynamic Link Architecture [5], [6] is an object-recognition system in which learned objects are represented by sparse graphs. Object recognition can be formulated as elastic graph matching. Another method for human face recognition is

- K.-M. Lam is with the Department of Electronic Engineering, The Hong Kong Polytechnic University, Hung Hom, Kowloon, Hong Kong.

E-mail:enkmlam@encserver.en.polyu.edu.hk.

- H. Yan is with the Department of Electrical Engineering, University of Sydney, NSW 2006, Australia.

Manuscript received 11 Mar. 1996; revised 13 May 1998. Recommended for acceptance by J. Daugman.

For information on obtaining reprints of this article, please send e-mail to: tpami@computer.org, and reference IEEECS Log Number 106860. by means of a similarity discriminant function (SDF) [7] of images. For each class of training image samples, an optimal projection axis maximizing the similarity among these training image samples is calculated. Then a hierarchical classifier in the optimal discriminant space is designed in order to recognize images. The singular values (SV) [8] feature vector is also used; it has some important properties of algebraic and geometric invariance, as well as an insensitivity to noise. All these methods require a long computing time since a face image is considered as a vector in a multidimensional space.

For the analytic approaches, a set of geometrical features from a face is computed. There are two main divisions of these approaches, based on feature vectors extracted from profile silhouettes [9], [10], [11], and from a frontal view of the face. In the profile analysis, a face profile is first reduced to outline curves, and then a number of fiducial marks are taken. These fiducial features are used to search for a candidate from a face database. In the frontal-view approaches, the positions of the different facial features and the face outline form a feature vector that represents a face. Chen et al. [12] used this approach for face recognition with a database of 12 faces. Brunelli et al. [13] achieved a 90 percent correct recognition rate by using geometric features. Kamel et al. [14] considered the perspective variations, and made use of the invariant properties of a number of interfeature point distances. With a set of 84 images, a recognition rate of 95 percent was obtained when the top four most likely matched faces were considered.

In our approach, we first use a new analytic method to select similar faces from a database. The positions of the different facial features and their outlines are located. A total of 15 feature points are chosen according to their significance, and the reliability of the detection. A head model is built in such a way that the rotation of the face can be 


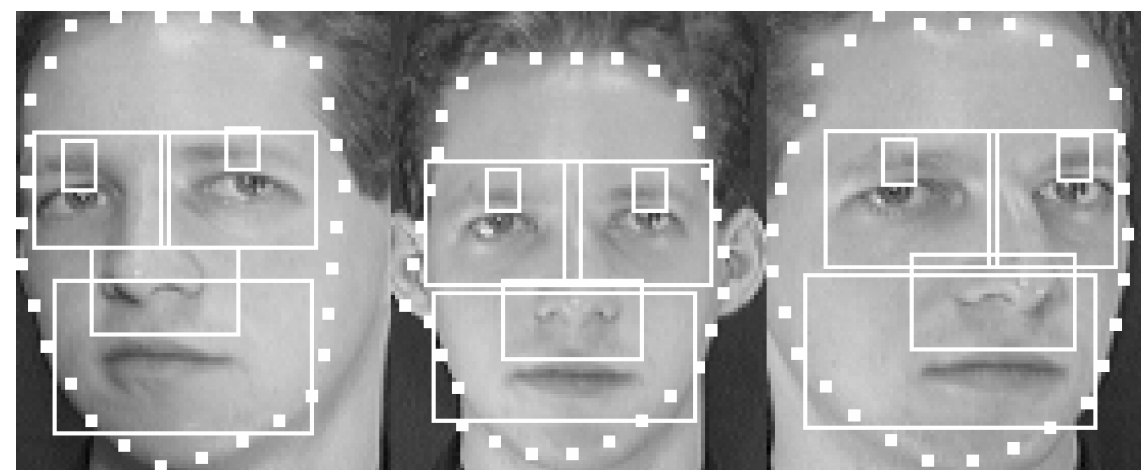

Fig. 1. Face boundary and the searching windows.

estimated by geometrical measurements. The feature points are adjusted to compensate for the effect of perspective variations. Only the similar faces in the database will be considered in the next step of face recognition. Correlations of the eyes, nose, and mouth are computed in the second step. These two parts are combined to form a complete face recognition system, which can achieve a high recognition rate under different perspective variations.

In Section 2 of this paper, we present the procedure for extracting different feature points in a face image. In Section 3, a head model is proposed, and the method of measuring its rotation is described. Based on the feature points, we propose the use of a similarity transform and pointmatching for face recognition in Section 3. Only those faces in the database similar to the input face are considered for the next step, which is computationally more time consuming. In this section, we describe the use of correlation for face recognition, which considers the geometrical distortion caused by head rotation. We present our experimental results on 160 test faces. Both the individual performance of the two steps and the combined performance are investigated. The recognition rates under different angles of observation are also provided.

\section{Locating the Facial Feature Points}

Locating facial features is an important step in human face recognition. In this paper, we locate 15 points on a human face and then use them for face recognition. These points are chosen based on their significance in representing a face, and the reliability of extracting them automatically. In the detection procedure, we first locate the face boundary using snakes. The approximate regions of the different facial features can be located using anthropometric standards. Among the feature points, six of them are located on the face boundary, four points for the eye corners, two for the mouth corners, two for the eyebrows, and one for the nose. The procedures for extracting all these feature points are described below.

\subsection{Locating the Face Boundary}

The extraction of the face boundary is performed as the first step in our feature extraction system. In order to extract and represent the face boundary efficiently and reliably, we make use of snakes, an active contour model [15]. The basic snake model is an energy-minimizing spline, which can be operated under the influence of internal contour forces, image forces and external constraint forces. A snake is represented as a parametric curve $\mathbf{v}(s)=(x(s), y(s))$, where the arc length, $s$, is a parameter. An energy functional of the snake is defined as

$$
\begin{aligned}
E_{\text {snake }}^{*} & =\int_{0}^{1} E_{\text {snake }}(\mathbf{v}(s)) d s \\
& =\int_{0}^{1} E_{\text {internal }}(\mathbf{v}(s))+E_{\text {image }}(\mathbf{v}(s))+E_{\text {constraint }}(\mathbf{v}(s)) d s
\end{aligned}
$$

where $E_{\text {internal }}$ represents the internal energy of the contour due to bending or discontinuities, $E_{\text {image }}$ is the energy of the image forces, and $E_{\text {constraint }}$ is the energy of the external constraint forces. The configuration of the snakes corresponds to the local minima of the energy functional.

We implement the snakes by means of a fast iterative method, namely, the fast greedy algorithm [16], [17] for the minimization of the energy functional of the active contour model. The energy functional to be minimized by this algorithm is

$$
E=\int\left(\alpha(s) E_{\text {continuity }}+\beta(s) E_{\text {curvature }}+\gamma(s) E_{\text {image }}\right) d s
$$

where $E_{\text {continuity, }}, E_{\text {curvature, }}$ and $E_{\text {image }}$ are the continuity force, curvature force and image force, respectively, and $\alpha, \beta$, and $\gamma$ are the respective weights of the three energy terms. The continuity force $E_{\text {continuity }}=|\bar{d}-| \mathbf{v}_{i}-\mathbf{v}_{i-1}||$ encourages even spacing of points, where $\bar{d}$ is the average distance between points. The curvature force $E_{\text {curvature }}=\left|\mathbf{v}_{i-1}-2 \mathbf{v}_{i}+\mathbf{v}_{i+1}\right|^{2}$ gives a reasonable and quick estimate of curvature. For a point with magnitude (mag), the image force, $E_{\text {imager }}$ is defined as $(\min -\operatorname{mag}) /(\max -\min )$, where $(\max )$ and $(\min )$ are the maximum and minimum gradient in each neighborhood. In this scheme, only a subset of the neighbors of a point is considered when computing the energy function in each iteration.

After the face boundary is located and measured, the approximate position of the mouth can be estimated. A searching window is then established for it. The reason for locating the mouth first among all the other facial features is that it can be detected and located reliably under wide perspective variations. Based on the face boundary and mouth position, other feature points can also be located reliably. The face boundary and the searching windows for the different facial features are illustrated in Fig. 1. 

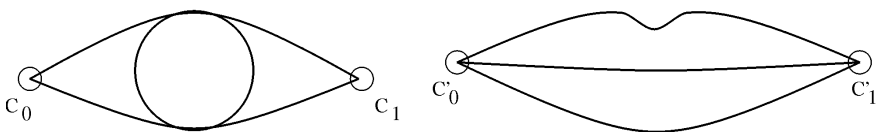

Fig. 2. Corners of the mouth and eyes.

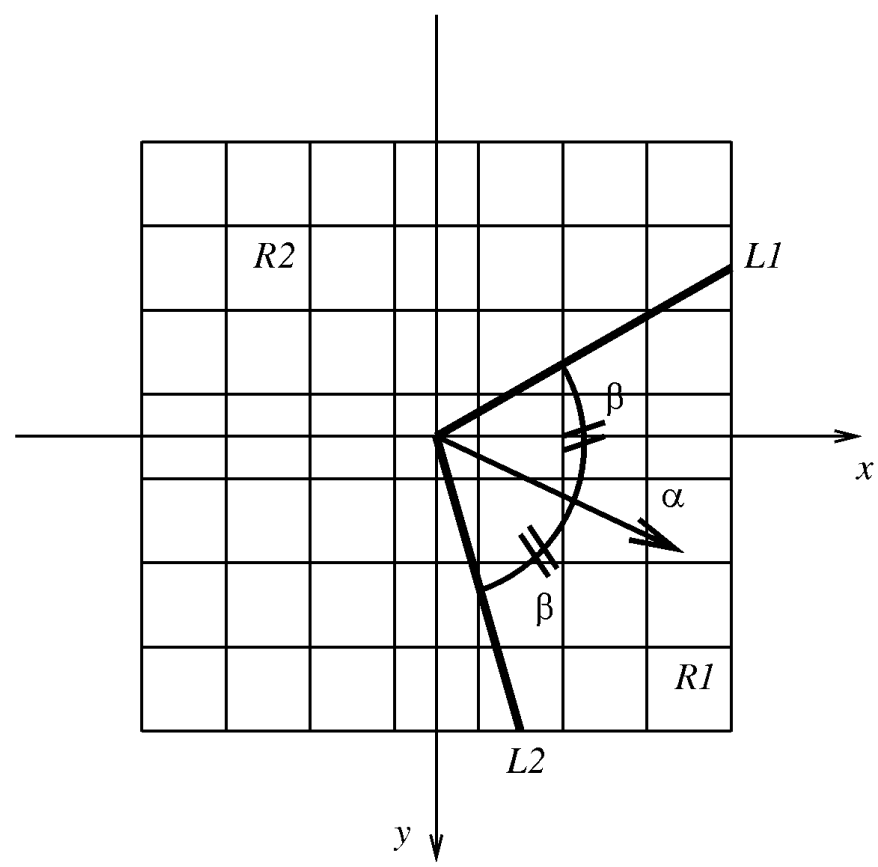

Fig. 3. Two regions, $R_{1}$ and $R_{2}$, bounded by edges $L_{1}$ and $L_{2}$.

\subsection{Detection of the Mouth and Eyes}

These two facial features are located by their respective two corners, as shown in Fig. 2. Corners are important feature points in shape description. Their positions are less susceptible to the changes of facial expressions. The corner detection scheme is based on that proposed by Xie et al. [18], which can provide information on corner orientations and angles in addition to corner locations. The procedures for identifying the different corner positions are described in [19], [20], which locate the different corner positions by using cost functions.

A corner is formed by the intersection of two lines. To classify a corner, the following properties are defined (with reference to Fig. 3):

1) The curvature $\beta$ is defined as the acute angle between the two lines.

2) The orientation $\alpha$ represents the orientation of the line which bisects the acute angle between the two lines.

3) The region dissimilarity, $\mathcal{D}$, is a measurement of the difference between the gray level averages in the two regions $R_{1}$ and $R_{2}$ bounded by the edges.

$D$ is defined as

$$
\mathcal{D}\left(R_{1}, R_{2}\right)=\frac{1}{n_{1}} \sum_{R_{1}} I(x, y)-\frac{1}{n_{2}} \sum_{R_{2}} I(x, y)
$$

where $I(x, y)$ is the gray level intensity of a pixel, and $n_{1}, n_{2}$ are the number of pixels in $R_{1}$ and $R_{2}$, respectively.
TABLE 1

Properties of the CORNERS OF THE EYE

\begin{tabular}{|c|c|c|c|}
\hline \hline & $\beta$ & $\alpha$ & $D$ \\
\hline \hline$C_{0}$ & $60^{\circ}-120^{\circ}$ & $-50^{\circ}-55^{\circ}$ & $\begin{array}{c}<-20 \text { for left eye } \\
<65 \text { for right eye }\end{array}$ \\
\hline$C_{1}$ & $60^{\circ}-120^{\circ}$ & $130^{\circ}-215^{\circ}$ & $\begin{array}{c}<65 \text { for left eye } \\
<-20 \text { for right eye }\end{array}$ \\
\hline
\end{tabular}

TABLE 2

PROPERTIES OF THE CORNERS OF THE MOUTH

\begin{tabular}{|c|c|c|c|}
\hline \hline & $\beta$ & $\alpha$ & $D$ \\
\hline \hline$C_{0}^{\prime}$ & $52.5^{\circ}-90^{\circ}$ & $-45^{\circ}-45^{\circ}$ & $<-10$ \\
\hline$C_{1}^{\prime}$ & $52.5^{\circ}-90^{\circ}$ & $135^{\circ}-225^{\circ}$ & $<-10$ \\
\hline \hline
\end{tabular}

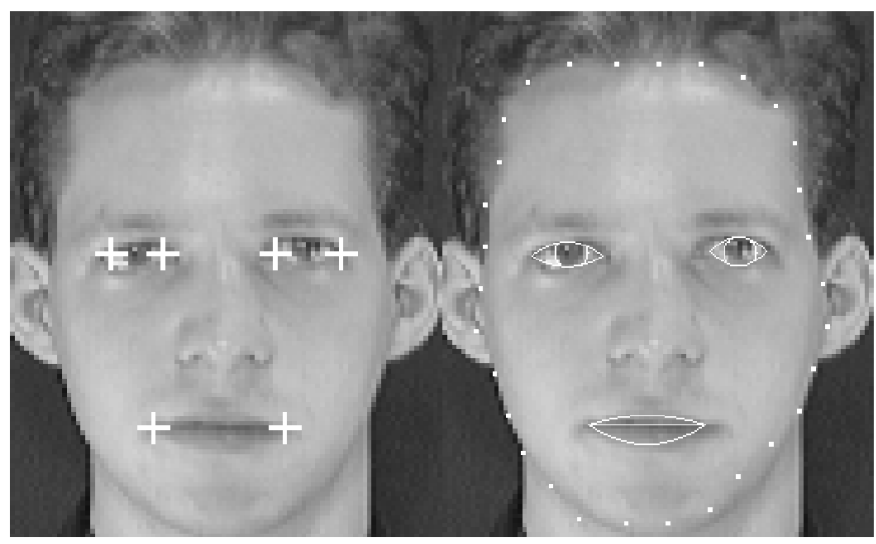

(a)

(b)

Fig. 4. (a) Corners detected at the mouth and eyes. (b) The corresponding shapes of the mouth and eyes, respectively.

The properties of each of the corners of the two facial features are shown in Table 1 and Table 2, respectively. A detected corner is classified as corner type $C_{i}$ if its properties satisfy those specified for $C_{i}$.

Fig. 4a shows examples of the corners for the mouth and eyes. Detection is based on the local information about a particular point. In order to obtain the accurate position of a corner, a global approach such as deformable template [21], [22] may be necessary. We use a new approach [23] based on deformable template and snakes, which can quickly extract the shapes of the eyes and mouth. An algorithm to detect whether a mouth is open or closed is described in [24], which is useful for face recognition, as explained in Section 4. Fig. 4b illustrates the shapes of the mouth and eyes based on our fast method.

\subsection{Locating the Eyebrows and Nose}

The locations of the eyebrows and nose are determined by means of integral projections. For the eyebrow, the position is along the line perpendicular to the line passing through the two eye corners at the irrespective midpoints. The nose is assumed to be on the symmetrical line of the face. How to set the symmetrical line accurately will be explained in Section 3.

Let $I(x, y)$ be our image. The horizontal integral projection of $I(x, y)$ in the $\left[x_{1}, x_{2}\right] \times\left[y_{1}, y_{2}\right]$ domain is defined as 


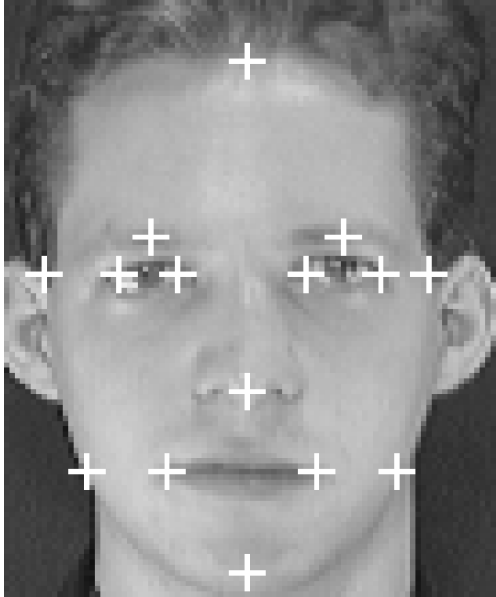

Fig. 5. The 15 feature points identified on the face.

$$
H(y)=\sum_{x=x_{1}}^{x_{2}} I(x, y)
$$

This technique was successfully used by T. Kanade [25] and R. Brunelli et al. [13] in their work on recognition of human faces. By measuring a local minimum from the projection, the vertical position of these two facial features can be located.

Fig. 5 illustrates the 15 feature points located on a face. These points are denoted as $p_{i}$, where $i=0, \cdots, 14$, with their coordinates represented as $\left(x_{p_{i}}, y_{p_{i}}, z_{p_{i}}\right)$. The $x$ - and $y$ coordinates are located, while the values of the $z$-coordinate are calculated according to the head model used. These points are described as follows:

$p_{0}, p_{1}, p_{2}, p_{3}$ the four eye corners from left to right,

$p_{4}, p_{5} \quad$ the left and right mouth corners,

$p_{6}, p_{7} \quad$ the positions of the left and right eyebrows,

$p_{8} \quad$ the position of the nose,

$p_{9}, p_{10} \quad$ the left and right interceptions of the face boundary and the least squared line passing through the four eye corners,

$p_{11}, p_{12} \quad$ the left and right interceptions of the face boundary and the line passing through the center of the mouth parallel to the line for the eyes,

$p_{13}, p_{14} \quad$ the top and bottom interceptions of the face boundary and the symmetrical line of the face.

\section{Head Models}

In most problems of face recognition, the face is considered as a flat, two-dimensional plane, and the effect of rotations are ignored. In this section, we propose a head model for the face. Our objectives here are to determine the rotation of the head, and then compensate for the effect of perspective variations on face recognition, using this model.

The head is basically considered as a cylindrical volume. The face, specifically, is less convex and close to a flat plane. To investigate the effect of the convexity of the face area on recognition, we have performed experiments of face recog-

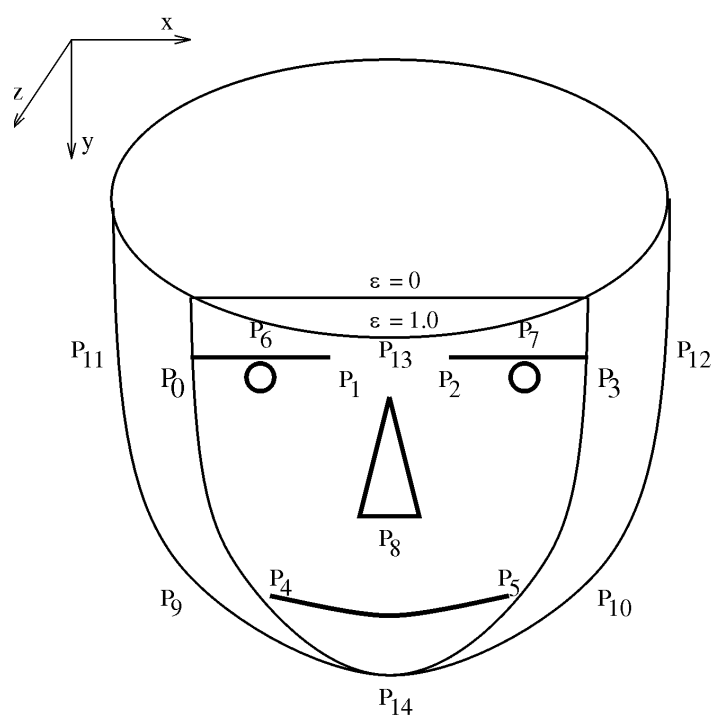

Fig. 6. The head model.

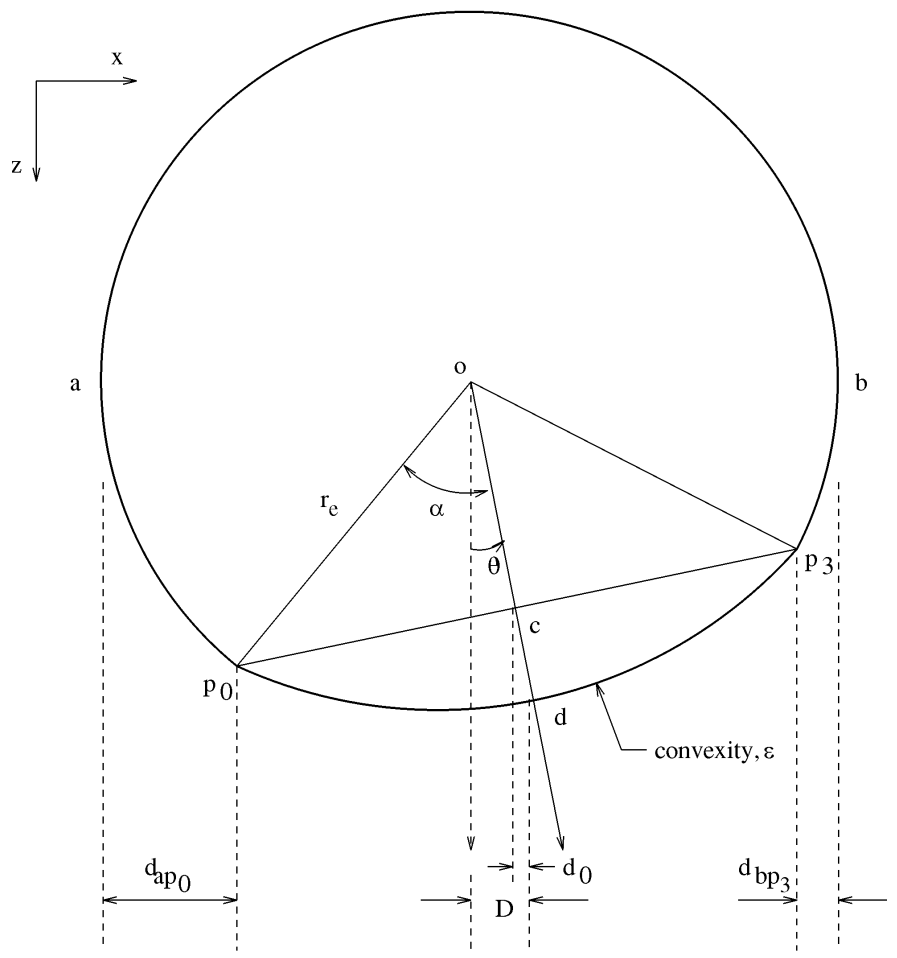

Fig. 7. The cross-section of the head model passing through the four eye corners.

nition with different convexity levels of the face. The head model is depicted in Fig. 6, with $\varepsilon$ defined as the convexity of the face. When $\varepsilon=0$, the face area becomes a flat plane, while the head is a cylinder if $\varepsilon=1$.

In this paper, we consider only the rotations on the $y$ and $z$-axes. The rotation on the $x$-axis is usually slight, so we assume that this rotation does not exist, or that it is too small to have any effect. In order to determine the rotation on the $y$-axis, we consider the cross-section of the head model passing through the four eye corners, as illustrated in Fig. 7. The angle of rotation on the $y$-axis is $\theta$, as shown. Based on the positions of the eye corners and the head 


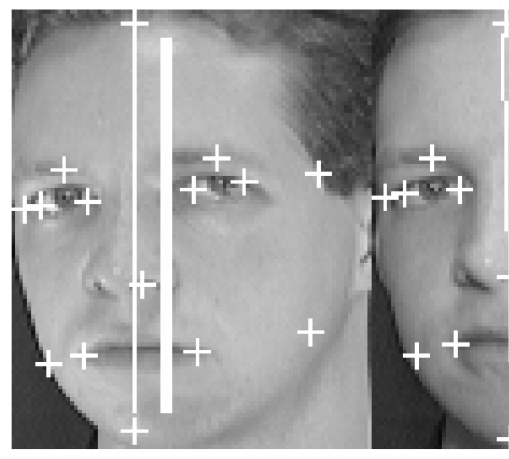

(a)

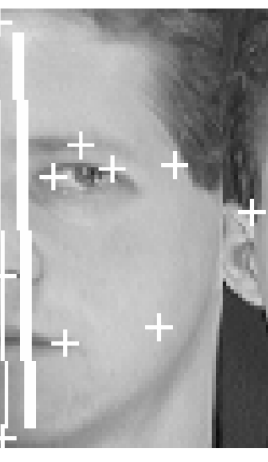

(b)

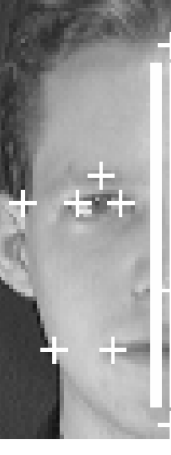

(c)

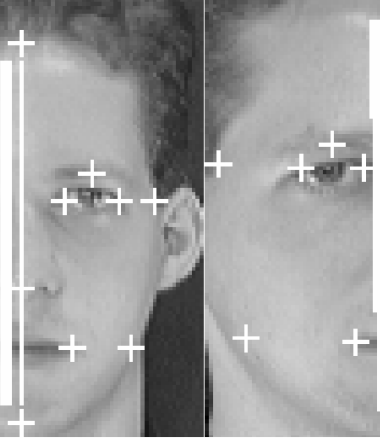

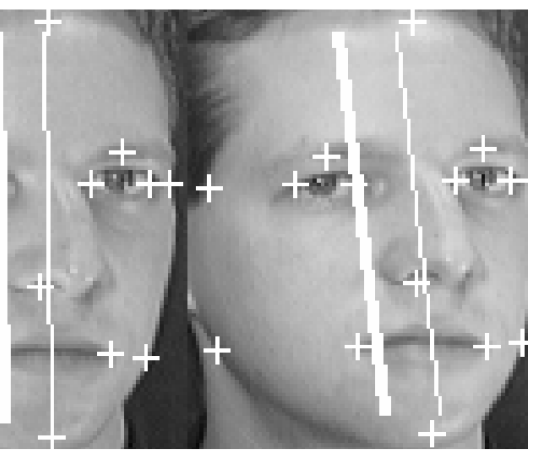

(d)

(e)

Fig. 8. The symmetrical line is represented by the thin line, while the center line by the thick line. The corresponding angle of rotation on the $y$-axis is illustrated. (a) $\theta=-13.0^{\circ}$. (b) $\theta=-9.1^{\circ}$. (c) $\theta=5.5^{\circ}$. (d) $\theta=15.9^{\circ}$. (e) $\theta=18.1^{\circ}$.

boundary, the distances $d_{a p_{0}}$ and $d_{b p_{3}}$, as shown in Fig. 7 can be measured. From the model, we have

$$
\begin{aligned}
& d_{a p_{0}}=r_{e}\{1-\sin (\alpha-\theta)\} \\
& d_{b p_{3}}=r_{e}\{1-\sin (\alpha+\theta)\}
\end{aligned}
$$

where $d_{i j}$ represents the distance from point $i$ to point $j, r_{e}$ is the radius of the head at the eye section, and $\alpha$ is the angle at the head center between the center of the face and the leftmost or the rightmost eye corner, respectively. By solving the above two equations, we have

$$
\begin{aligned}
& \alpha=\frac{1}{2}\left\{\sin ^{-1}\left(1-\frac{d_{b p_{3}}}{r_{e}}\right)+\sin ^{-1}\left(1-\frac{d_{a p_{0}}}{r_{e}}\right)\right\} \\
& \theta=\theta_{1}=\frac{1}{2}\left\{\sin ^{-1}\left(1-\frac{d_{b p_{3}}}{r_{e}}\right)-\sin ^{-1}\left(1-\frac{d_{a p_{0}}}{r_{e}}\right)\right\}
\end{aligned}
$$

The angle $\theta$ can also be calculated by measuring the distance, $D$, between the symmetrical line of the face and the center line of the face on the section. The symmetrical line of the face is a line which divides the face vertically into two equal sides. The position of the point of the symmetrical line depends on the convexity, $\varepsilon$, of the face. The protrusion of the face, $d_{c d}$, is

$$
d_{c d}=\varepsilon \cdot r_{e}(1-\cos \alpha)
$$

where point $c$ is the midpoint of the eye corners $p_{0}$ and $p_{3}$, while $d$ is a point on the symmetrical line of the face. The horizontal distance between these two points is given by

$$
d_{0}=d_{c d} \cdot \sin \theta_{1}
$$

The coordinates of the point $d$ is therefore given by

$$
x_{d}=\frac{x_{p_{0}}+x_{p_{3}}}{2}+d_{0}, \quad y_{d}=\frac{y_{p_{0}}+y_{p_{3}}}{2}
$$

Similarly, the point on the symmetrical line passing through the mid-section of the mouth can be located. By joining these two points, as shown by the thin line in Fig. 8, the symmetrical line of the face is located. The center line of the face is defined as the line parallel to the symmetrical line of the face, and its position exhibits the least squared error in relation to the points of the face boundary. The equation of the center line is represented as follows:

$$
y=m \cdot x+c^{\prime}
$$

where $m$ is equal to the gradient of the symmetrical line of the face. If we denote $\left(x_{i}, y_{i}\right)$ as the points of the snakes, we have

$$
c^{\prime}=\left\{\begin{array}{l}
\frac{\sum_{i} x_{i}}{n}, \quad \text { if } m=\infty \\
\frac{\sum_{i} y_{i}-m \cdot \sum_{i} x_{i}}{n}, \quad \text { otherwise }
\end{array}\right.
$$

where $n$ is the number of points on the snakes. The center line is shown as the thick line in Fig. 8. By measuring the distance, $D$, between these two lines, the angle $\theta$ can be estimated as follows:

$$
\begin{gathered}
d_{o d}=r_{e} \cdot \cos \alpha+\varepsilon \cdot r_{e}(1-\cos \alpha) \\
\theta=\theta_{2}=\sin ^{-1}\left(\frac{D}{d_{o d}}\right)
\end{gathered}
$$

Therefore, the angle $\theta$ is given by

$$
\theta=\frac{\theta_{1}+\theta_{2}}{2}
$$

In Fig. 8, the angles of rotation on the $y$-axis are also illustrated. The angle of rotation, $\beta$, on the $z$-axis can be estimated by the gradient of the best-fitting line passing through the four eye corners. By knowing these two angles, transformation can take place, which compensates for the effect of rotations.

\section{4 face Recognition}

In this section, we present two methods of face recognition and investigate their individual and combined performances. The first method is based on the information concerning where the facial features are. The 15 feature points described in Section 2 are used to represent a face. The positions of these points are adjusted according to the angles of rotation, as described in Section 3. The point set for an input face is then compared with the point sets of the faces in a database by point-matching. Faces similar to the input are chosen from the database and passed on for the second 
recognition process which measures the difference in context for the different facial features between the input and the database. Three windows, namely, the eyes and eyebrows window, the nose window, and the mouth window, are established for each face. Before giving the details of these two methods of face recognition, we will first describe the database used in the experiment and also discuss the evaluation of a recognition system.

\subsection{Database}

The database used in this paper was created by the Olivetti Research Laboratory in Cambridge, UK. There are 10 different images of 40 distinct faces. In our experiment, we chose a face with an upright frontal view for each of the 40 faces as our database. In other words, each person has a single frontal view in our database. Among the rest of the faces, we selected 160 images as a testing set. About half of the faces are upright and have a small rotation on the $y$-axis. The other half of the faces show different amounts of perspective variations. In the following sections, we will measure the performance of the two recognition methods with different amounts of perspective variations.

\subsection{Evaluation of Performance for Recognition}

The recognition rate is always the index used to evaluate the performance of a recognition system. For face recognition and some other applications, a list of the objects in the database is sequenced according to their similarity to the input image. The object that best matches the input is placed on top of the list, while the least matched one is at the bottom. It is useful to measure the probability of the input at different positions on the list. For a database of $N$ objects, we define the cumulative recognition rate, $P(n)$, as follows:

$$
\begin{aligned}
P(n)= & \text { the probability of the input being chosen } \\
& \text { within the first top } n \text { objects on the list } \\
= & \sum_{i=0}^{n} p(i)
\end{aligned}
$$

where $n=0, \cdots, N-1$, and $p(i)$ is the probability of the correct object being placed at the $i$ th position on the list. The top of the list is at position 0 , while the last item is said to be at position $N-1$. This parameter not only gives the recognition rate by $P(0)$, but also provides the recognition rates if more objects on the list are considered. A list of similar faces, rather than the most matched one only, is usually displayed in face recognition.

The other parameter $R$, called the overall recognition index, is defined as follows to describe the overall performance of recognition:

$$
R=1-\frac{1}{N_{t}} \sum_{i=1}^{N_{t}} \frac{r(i)}{N-1}
$$

where $N_{t}$ is the number of test images and $r(i)=0, \cdots, N-1$ is the position of the correct object on the list. This parameter is equal to one if all the testing images are identified correctly and is zero if they are all recognized as the least matched.
We believe that these two parameters can provide more information about recognition performance and offer an effective means to compare the overall performance of different recognition systems.

\subsection{Face Recognition by Point-Matching}

In this section, we present a point-matching scheme for face recognition which is independent of the rotations of the head. In Section 2, we have described the procedures for extracting the 15 feature points, $p_{0}, \cdots, p_{14}$, automatically. In Section 3, we have presented the determination of the angles of rotation, $\theta$ and $\beta$. The point set of an input image is adjusted by a transformation matrix to compensate for the rotation and then compared to the faces in a database by means of a similarity transform.

\subsubsection{Transformation Matrix}

The following rotation matrix can be used to compensate for the effect of rotation.

$$
A_{\beta \theta}=\left(\begin{array}{ccc}
\cos \theta \cos \beta & \sin \beta \sin \beta & -\cos \beta \sin \theta \\
-\sin \beta \cos \theta & \cos \beta & \sin \beta \sin \theta
\end{array}\right)
$$

The origin of the coordinate system is translated to the point $O$ (with reference to Fig. 7). The angle $\beta$ is given by

$$
\beta=\sin ^{-1} \frac{y_{p_{3}}-y_{p_{0}}}{\left\|p_{3}-p_{0}\right\|}
$$

This transformation will only apply to the points $p_{0}$ to $p_{7}$. Points $p_{9}$ to $p_{12}$ are on the face boundary. The distances $d_{p_{9} p_{10}}$ and $d_{p_{11} p_{12}}$ are assumed to remain unchanged, but their positions are adjusted in such a way that the respective centers of the two pairs lie on the symmetrical line. The lines passing through these two point pairs are parallel to the line passing through the four eye corners after transformation. The new symmetrical line is obtained by joining the midpoints of the eye corners and mouth corners. $p_{8}, p_{13}$, and $p_{14}$ are placed on the symmetrical line of the face, and their respective distances from the line of eye corners remain unchanged.

For the transformation, the $z$-coordinates of the points are also required in the computation. These values can be approximated according to the head model. The $z$-coordinates of the leftmost and rightmost eye corners, $p_{0}$ and $p_{3}$, and the two mouth corners, $p_{6}$ and $p_{7}$, are given as follows:

$$
\begin{aligned}
& z_{p_{0}}=r_{e} \cdot \cos (\alpha-\theta) \\
& z_{p_{3}}=r_{e} \cdot \cos (\alpha+\theta) \\
& z_{p_{4}}=r_{m} \cdot \cos (\alpha-\theta) \\
& z_{p_{5}}=r_{m} \cdot \cos (\alpha+\theta)
\end{aligned}
$$

where $r_{e}$ and $r_{m}$ are the radii of the head at the eye and mouth section, respectively. To calculate $z_{p_{1}}, z_{p_{2}}, z_{p_{6}}$, and $z_{p_{7}}$, we consider the arc formed by the points $p_{0}, d$, and $p_{3}$, which is also a part of a circle. From these three points, the center, $\left(x_{f}, z_{f}\right)$, and radius, $r_{f}$, of this circle can be computed. The $z$-coordinates of these points are then 


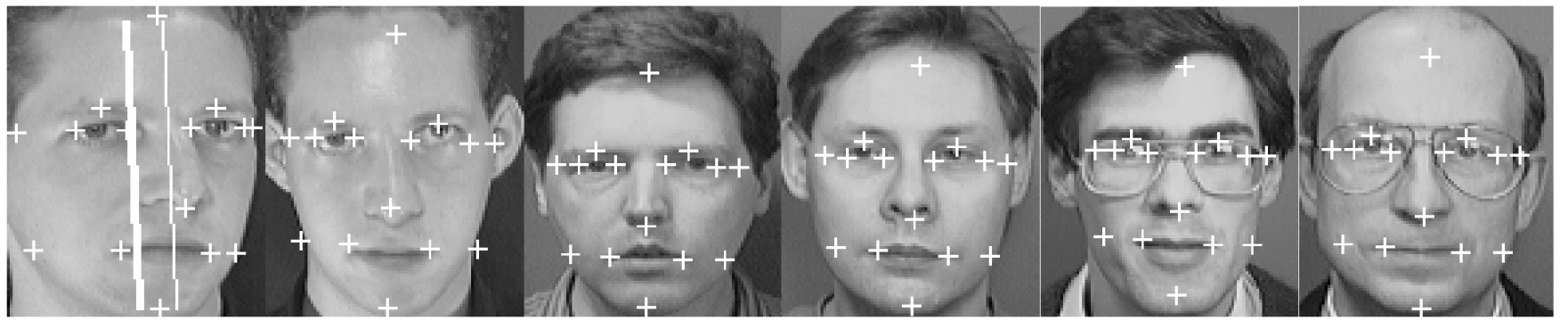

(a)

(b)

(c)

(d)

(e)

(f)

Fig. 9. The 2D point-matching of an input face to the database. The first face is the input and the others are faces in the database. The second face is the same person as the input. The corresponding $D_{w}$ s are: (b) $D_{w}=0.081$. (c) $D_{w}=0.097$. (d) $D_{w}=0.131$. (e) $D_{w}=0.155$. (f) $D_{w}=0.166$.

$$
z_{p_{i}}=\sqrt{r_{f}^{2}-\left(x_{p_{i}}-x_{f}\right)^{2}}+z_{f}, \quad \text { where } i=1,2,6,7 \text {. }
$$

When the convexity $\varepsilon$ of the face is set to zero, the front of the face becomes a flat plane and the above equation is no longer valid. In this case, the $z$-coordinates are given by

$$
z_{p_{i}}=z_{p_{0}}-\left(x_{p_{i}}-x_{p_{0}}\right) \cdot \tan \theta, \quad \text { where } i=1,2,6,7 \text {. }
$$

The transformed point set is denoted as $P_{i}, i=0, \cdots, 14$, which is then used for recognizing faces by $2 \mathrm{D}$ pointmatching.

\subsubsection{Recognition by Matching 2D Point Sets}

In the previous section, a set of 15 compensated feature points $\left\{\left(x_{P_{i}}, y_{P_{i}}\right)\right\}_{i=0}^{14}$ were located for the input image. Similarly, a set of 15 feature points, denoted as $\left\{\left(x_{Q_{i}}, y_{Q_{i}}\right)\right\}_{i=0}^{14}$, were also located for each face in the database. These point sets are represented by a $2 \times 15$ matrix, which are denoted as $\mathbf{P}$ and $\mathbf{Q}$, respectively. The $i$ th column of the matrix represents the $x y$-coordinates of the point $P_{i}$ of the corresponding image. To compare the two point sets is equivalent to measuring the matrices $\mathbf{P}$ and $\mathbf{Q}$. The metric usually used is the Frobenius norm of the difference matrix, which is the same as the Euclidean distance between points in the images. In our case, because the 15 feature points have different degrees of significance and reliability for representing a face, the weighted Euclidean distance, $D_{w}$, is used to compare two point sets.

$$
\begin{aligned}
D_{w} & =\sum_{i=0}^{14} \kappa_{i}\left\|P_{i}-Q_{i}\right\|^{2} \\
& =\sum_{i=0}^{14} \kappa_{i}\left\{\left(x_{P_{i}}-x_{Q_{i}}\right)^{2}+\left(y_{P_{i}}-y_{Q_{i}}\right)^{2}\right\}
\end{aligned}
$$

where $\kappa_{i}$ is the weighting factor for $P_{i}$ and $Q_{i}$.

To measure the difference between two point sets, two operations, namely normalization and point alignment, are necessary to remove differences due to irrelevant effects between the two faces. We use the similarity measure proposed by Werman et al. [26] to normalize and align the two point sets before performing the comparison. First of all, each point set is translated to its centroid, so that its first moment is zero. As the input face has to align with each face in the da- tabase, we select a subset of the point sets for alignment in order to reduce the required computation. Three points, the centers of the two eyes and the center of the mouth, are chosen from each point set for alignment. These two point sets are denoted as $\mathcal{P}$ and $\mathcal{Q}$ respectively, such that

$$
\begin{array}{ll}
x_{\mathcal{P}_{0}}=\frac{x_{P_{0}}+x_{P_{1}}}{2} & y_{\mathcal{P}_{0}}=\frac{y_{P_{0}}+y_{P_{1}}}{2} \\
x_{\mathcal{P}_{1}}=\frac{x_{P_{2}}+x_{P_{3}}}{2} & y_{\mathcal{P}_{1}}=\frac{y_{P_{2}}+y_{P_{3}}}{2} \\
x_{\mathcal{P}_{2}}=\frac{x_{P_{4}}+x_{P_{5}}}{2} & y_{\mathcal{P}_{2}}=\frac{y_{P_{4}}+y_{P_{5}}}{2}
\end{array}
$$

Scale normalization is then applied to the point sets as follows:

$$
\begin{array}{ll}
\mathbf{P}^{\prime}=\frac{\mathbf{P}}{\|\mathcal{P}\|}, & \mathbf{Q}^{\prime}=\frac{\mathbf{Q}}{\|\mathcal{Q}\|} \\
\mathcal{P}^{\prime}=\frac{\mathcal{P}}{\|\mathcal{P}\|}, & \mathcal{Q}^{\prime}=\frac{\mathcal{Q}}{\|\mathcal{Q}\|}
\end{array}
$$

The point set $\mathcal{P}^{\prime}$ is aligned with $\mathcal{Q}^{\prime}$ using a scaled rotation

$$
\mathcal{P}^{\prime \prime}=s R \cdot \mathcal{P}^{\prime}
$$

where $s$ is a scalar and $R$ is a $2 \times 2$ rotation matrix

$$
R=\left(\begin{array}{cc}
\cos \mu & \sin \mu \\
-\sin \mu & \cos \mu
\end{array}\right)
$$

where $\mu$ is the angle of rotation for alignment. The respective values of $s$ and $\mu$ are chosen to minimize $\left\|s R \cdot P^{\prime}-\mathcal{Q}^{\prime}\right\|$. These two values are shown in [24] and are given as follows:

$$
\begin{aligned}
s & =\sqrt{r t^{2}\left[\mathcal{P}^{\prime}\left(\mathcal{Q}^{\prime}\right)^{T}\right]+t r^{2}\left[\mathcal{P}^{\prime}\left(\mathcal{Q}^{\prime}\right)^{T}\right]} \\
\tan \mu & =\frac{r t\left[\mathcal{P}^{\prime}\left(\mathcal{Q}^{\prime}\right)^{T}\right]}{\operatorname{tr}\left[\mathcal{P}^{\prime}\left(\mathcal{Q}^{\prime}\right)^{T}\right]}
\end{aligned}
$$

The 2D alignment transformation is therefore

$$
s R=\left(\begin{array}{cc}
\operatorname{tr}\left[\mathcal{P}^{\prime}\left(\mathcal{Q}^{\prime}\right)^{T}\right] & r t\left[\mathcal{P}^{\prime}\left(\mathcal{Q}^{\prime}\right)^{T}\right] \\
-r t\left[\mathcal{P}^{\prime}\left(\mathcal{Q}^{\prime}\right)^{T}\right] & \operatorname{tr}\left[\mathcal{P}^{\prime}\left(\mathcal{Q}^{\prime}\right)^{T}\right]
\end{array}\right)
$$

Based on the above procedures, the point set of the input face is normalized, aligned and compared with the normalized point sets of faces in the database. Fig. 9 shows an input face matching its point set to five faces in the database. The corresponding $D_{w}$ s are also illustrated. 


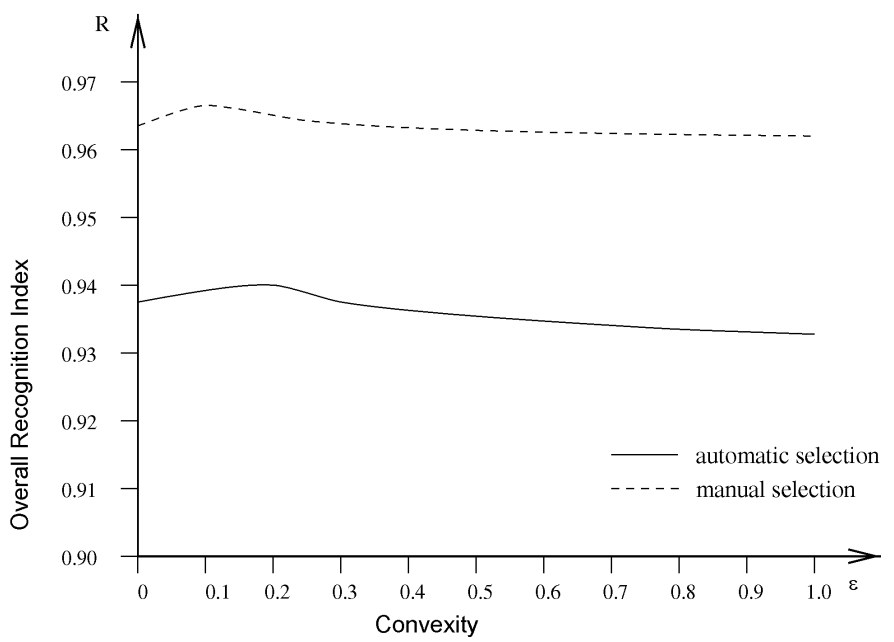

Fig. 10. The effect of the convexity, $\varepsilon$, of the face on the overall recognition indices, $R$.

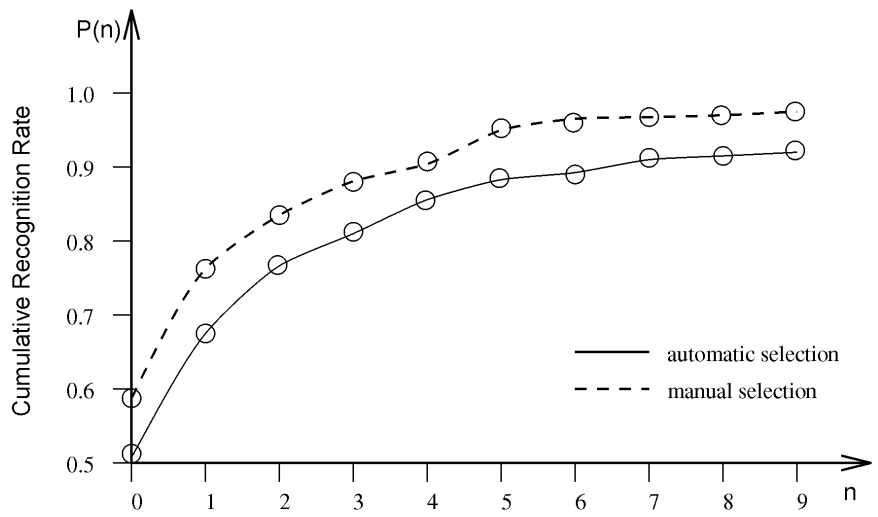

Fig. 11. The cumulative recognition rates, $P(n)$, for $\varepsilon=0.15$ with manual and automatic selection procedures of feature points.

\subsubsection{Experiments}

In this section, the performance of the point-matching scheme regarding the head model is evaluated. In our system, the feature points are detected automatically. However, due to the poor illumination conditions and the existence of glasses and a moustache in some of the images, some feature points may sometimes not be located accurately. Therefore, the performance of the point-matching scheme with automatic feature point selection procedure is compared with using manual selection procedure, which locates the feature points by a human. We first investigate the effect of the convexity, $\varepsilon$, of the face on the recognition rates. Fig. 10 shows the overall recognition indices, $R$, for different values of $\varepsilon$. It can be seen that the best performance is achieved when $\varepsilon \approx 0.20$ for the automatic selection procedure, while $\varepsilon \approx 0.10$ for the manual selection procedure. In the rest of this paper, we adopt $\varepsilon=0.15$ for the head model in the experiments. The corresponding cumulative recognition rates for $\varepsilon=0.15$ are depicted in Fig. 11. It can be seen that the overall recognition rate is over 50 percent, and is increased to over 80 percent by considering the first four best matched faces.

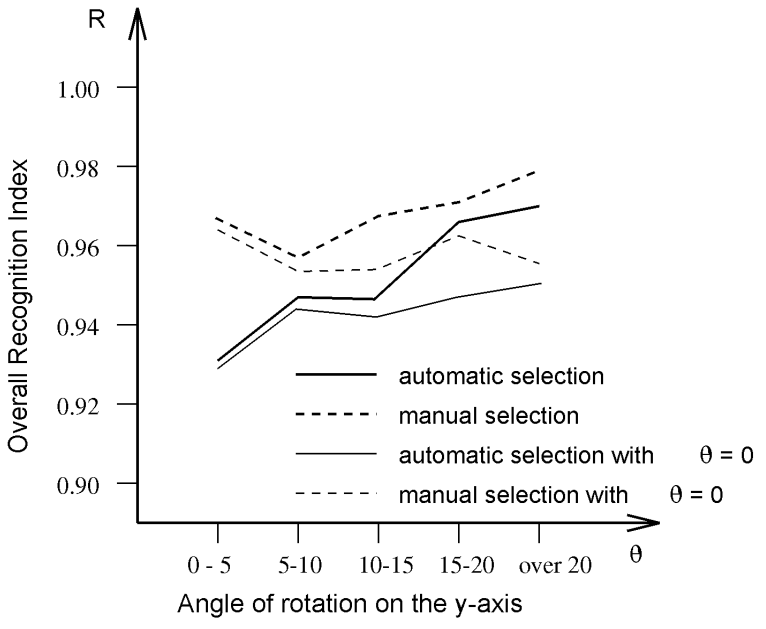

Fig. 12. The overall recognition indices, $R$, for different values of $\theta$ with both selection procedures of the feature points, and with and without compensating for the effect of $\theta$.

We compare the performance of the scheme regarding automatic and manual selection procedures of the feature points. In order to investigate the effect of the rotation, $\theta$, as well, we illustrate the overall recognition indices for different values of $\theta$ with both selection procedures of the feature points, as shown in Fig. 12. The corresponding cumulative recognition rates, $P(n)$, at different angles of rotation are illustrated in Fig. 13. Furthermore, we also compare the results with and without compensating for the effect of the rotation, $\theta$. The performance with compensation is insensitive to rotation, while the value, $R$, decreases with an increased rotation angle, $\theta$, if compensation is not considered, as illustrated in Fig. 12. The results are obtained by just setting $\theta=0$ in (16).

This recognition step can be used to select from the database the faces similar to the input. In our system, the second step will perform recognition by correlation, which is computationally intensive. By cutting the number of candidates to be passed to this second stage, the overall computation is greatly reduced. A threshold, $\mathcal{D}$, is chosen. A candidate in the database will not be considered for further processing if its value, $D_{w}$, is larger than $\mathcal{D}$. If $\mathcal{D}$ is set too high, all the candidates will be passed. However, if it is too low, then the correct candidate may be rejected at this stage. Fig. 14 shows the effect of $\mathcal{D}$ on the average number of candidates passed to the next stage, and the corresponding number of correct candidates being rejected.

\subsection{Recognition by Correlation}

In the previous sections, we located the facial features and identified a face based on these feature locations. Only those faces in the database which are similar to or have a similar configuration to the input are considered for further processing. Correlation [25] has been proved to be an effective method for recognition, but this operation is computationally intensive. Correlation is applied to compare the faces passed from the point-matching step, as well as the contents of the regions of the facial features. 


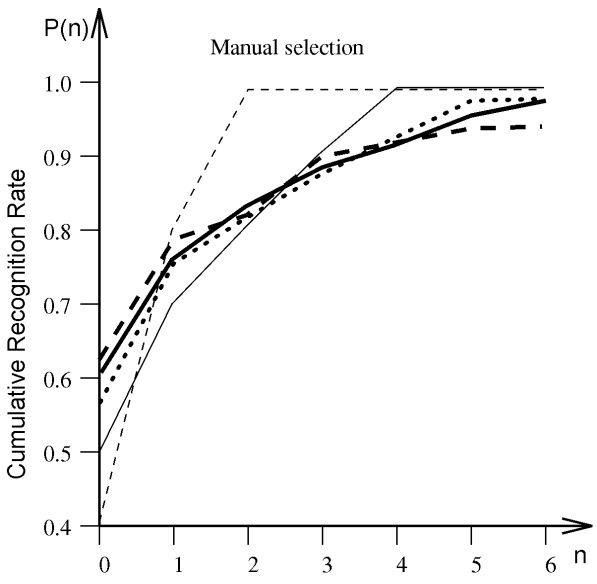

(a)

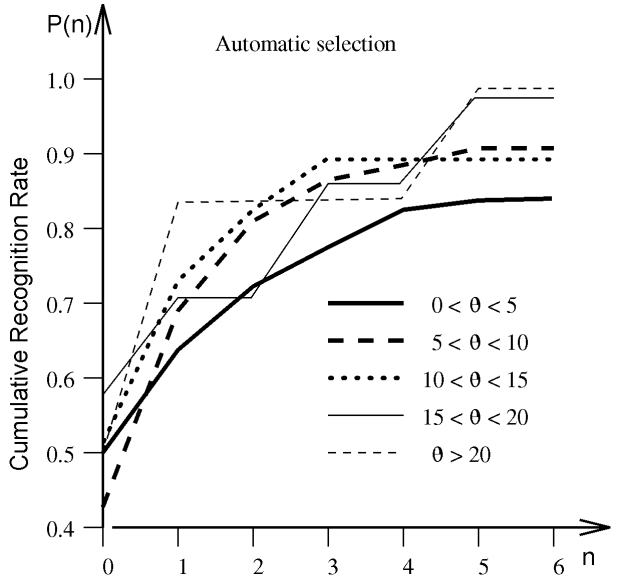

(b)

Fig. 13. The cumulative recognition rates, $P(n)$, at different angles of rotation with (a) manual, and (b) automatic selection procedures of the feature points.

Three windows-the eyes and eyebrows window, the nose window, and the mouth window-are set up for each face. Those windows of the input are compared to the corresponding windows of each face in the database. The sizes of the eyes and eyebrows window, the nose window and the mouth window are $60 \times 22,28 \times 22$, and $40 \times 17$, respectively. Correlation is affected by the angle of rotation, $\theta$, so transformation and alignment are performed on the images inside the windows.

\subsubsection{Correlation}

Correlation is a measure of the similarity of two images. A common method of estimating the similarity of two images is by means of the Euclidean distance. However, this approach is affected by large errors. To cope with the high sensitivity to noise exhibited by the Euclidean distance approach, Brunelli et al. [27] proposed other similarity measures based on the $L_{1}$ norm, which exhibits a lower sensitivity to noise. We chose the following similarity measure:

$$
g(\mathbf{x}, \mathbf{y})=1-\frac{\sum_{i}\left|x_{i}-y_{i}\right|}{\sum_{i}\left(\left|x_{i}\right|+\left|y_{i}\right|\right)}
$$

where $\mathbf{x}$ and $\mathbf{y}$ are the input image and the image in the database, respectively. For this similarity measure, $\Sigma_{i}\left|y_{i}\right|$ can be precomputed and is stored with the image in the database, while the term $\Sigma_{i}\left|x_{i}\right|$ is computed only once for the input. The computation for $g(\mathbf{x}, \mathbf{y})$, therefore comes mainly from the term $\Sigma_{i}\left|x_{i}-y_{i}\right|$, which is much lower than the other similarity measures.

\subsubsection{The Eyes and Eyebrows Window}

The size of the eyes and eyebrows window is $60 \times 22$. Each image is transformed and aligned in such a way that the two eye corners, $p_{0}$ and $p_{3}$, in the image domain are placed at $(5,15)$ and $(55,15)$, respectively, in the window domain. These two points are denoted as $p_{0}^{\prime}$ and $p_{3}^{\prime}$, respectively. The center of the image, $d$ (with reference to Fig. 7), is transformed and aligned to $(30,15)$ in the window and is de- noted as $d^{\prime}$. We translate the coordinate systems to $(30,15)$ so that the coordinates of the two eye corners become $(-25,0)$ and $(25,0)$, respectively. In the transformation, the points are considered to be $3 \mathrm{D}$, and so their $z$-coordinates have to be determined. The radius of the head at the transformed eye section is

$$
r_{e}^{\prime}=\frac{50 \cdot r_{e} \cdot \cos \theta}{\left\|p_{3}-p_{1}\right\|}
$$

The $z$-coordinates of the points $p_{0}^{\prime}, p_{3}^{\prime}$, and $d^{\prime}$ are

$$
\begin{aligned}
& z_{p_{0}^{\prime}}=z_{p_{3}^{\prime}}=r_{e}^{\prime} \cdot \cos \alpha \\
& z_{d^{\prime}}=\varepsilon \cdot r_{e}^{\prime}+(1-\varepsilon) \cdot r_{e}^{\prime} \cdot \cos \alpha
\end{aligned}
$$

The coordinates of the points in the image domain are obtained by transforming the points in the window domain using the following transformation matrix.

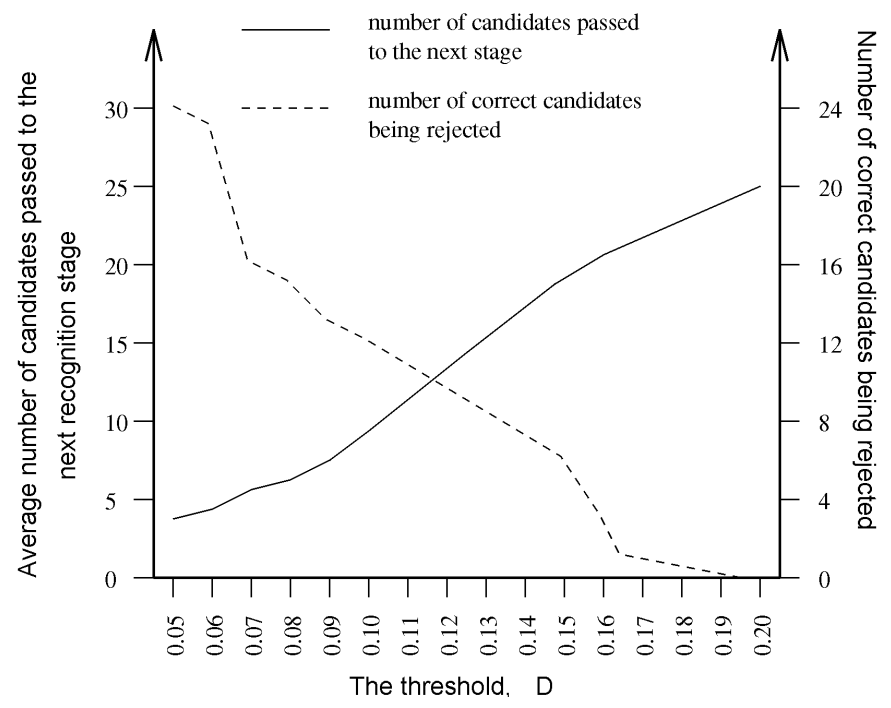

Fig. 14. The effect of $\mathcal{D}$ on (a) the average number of candidates passed to the next stage, and (b) the number of correct candidates being rejected. 


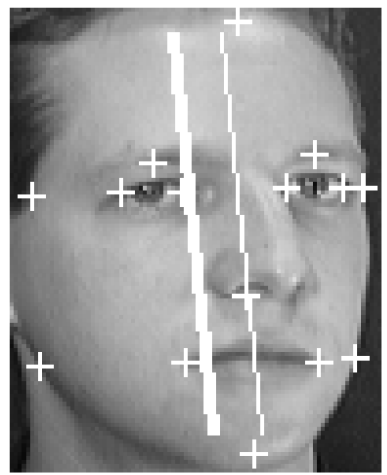

(a)
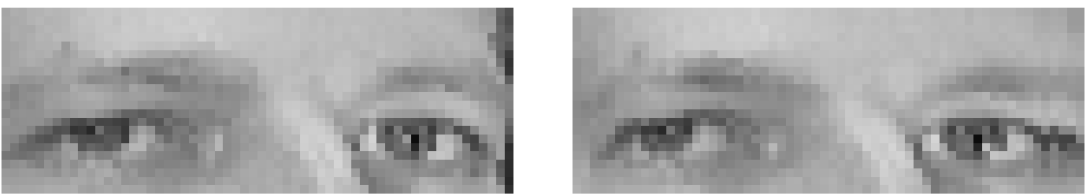

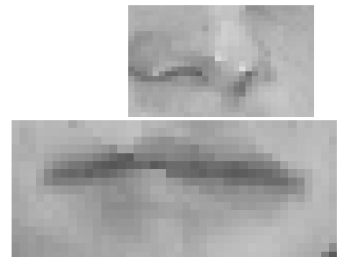

(b)

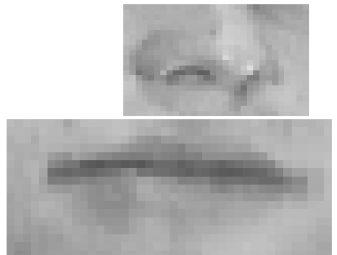

(c)

Fig. 15. (a) The face with feature points marked. (b) The three windows of facial features without using the transformation, $g_{e}=0.933, g_{n}=0.951$, $g_{m}=0.931$. (c) With using the transformation, $g_{e}=0.938, g_{n}=0.954, g_{m}=0.933 . g_{e}, g_{n}$, and $g_{m}$, are the correlation values for the eyes and eyebrows window, the nose window, and the mouth window, respectively.

$$
A_{\theta \beta}^{\prime}=\left(\begin{array}{ccc}
\cos \theta \cos \beta & -\cos \theta \cos \beta & \sin \theta \\
\sin \beta & \cos \beta & 0
\end{array}\right)
$$

To align the two eye corners, the transformed coordinates are scaled with a scale given by

$$
r=\frac{x_{p_{3}}-x_{p_{0}}}{50 \cos \theta \cos \beta}
$$

The overall transformation is therefore

$$
\left(\begin{array}{l}
x \\
y
\end{array}\right)=r A_{\theta \beta}^{\prime}\left(\begin{array}{l}
x^{\prime} \\
y^{\prime} \\
z^{\prime}
\end{array}\right)+\left(\begin{array}{l}
x_{d^{\prime}} \\
y_{d^{\prime}}
\end{array}\right)
$$

where

$$
x_{d^{\prime}}=\frac{x_{p_{0}}+x_{p_{3}}}{2}
$$

and

$$
y_{d^{\prime}}=\frac{y_{p_{0}}+y_{p_{3}}}{2}
$$

The $z^{\prime}$ is determined according to whether the point is in the face region or not. In the face region, this cross section is a part of a circle and its $z$-coordinate is

$$
z_{f}^{\prime}=\frac{25^{2}+z_{p_{0}^{\prime}}^{2}-z_{d^{\prime}}^{2}}{2\left(z_{p_{0}^{\prime}}-z_{d^{\prime}}\right)}
$$

The $z$-coordinate of a point is then given by

$$
z^{\prime}=\sqrt{\left(z_{d^{\prime}}-z_{f}^{\prime}\right)^{2}-x^{\prime 2}}+z_{f}^{\prime}
$$

When outside the face region, the $z$-coordinate is

$$
z^{\prime}=\sqrt{z_{d^{\prime}}^{2}-x^{\prime 2}}
$$

Fig. 15 illustrates the eyes and eyebrows window both with and without using the transformation. The corresponding correlations are also shown at the bottom of each window. It is obvious that the correlation value is increased by the transformation. This operation can give an approximate upright frontal view of this part of the face.

\subsubsection{The Nose Window and the Mouth Window}

The nose and mouth images are transformed and aligned in the same way as the eyes and eyebrows image. The size of the nose window is $28 \times 22$. The coordinates $(14,15)$ are selected as the center, which is aligned to the point $p_{8}$. The scale used for the nose image is the same as the eyes and eyebrows image. The size of the mouth window is $40 \times 17$, with the center at $(20,7)$. The two mouth corners, $p_{4}$ and $p_{5}$, are used to align the mouth image. Fig. 15 also illustrates the transformed nose and mouth windows, and their corresponding values of correlation are shown.

\subsubsection{Experiments}

The performance of correlation for image matching is investigated for the three windows. The overall recognition 


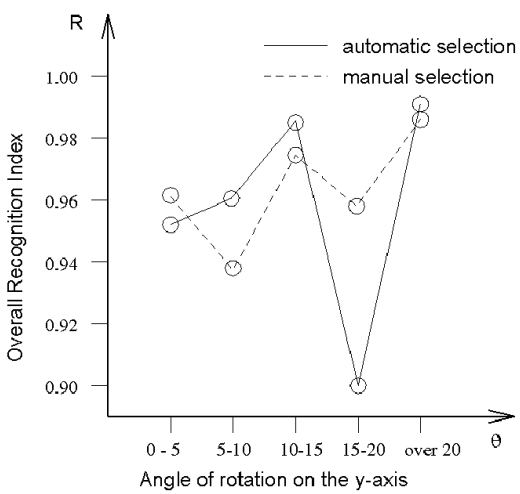

(a)

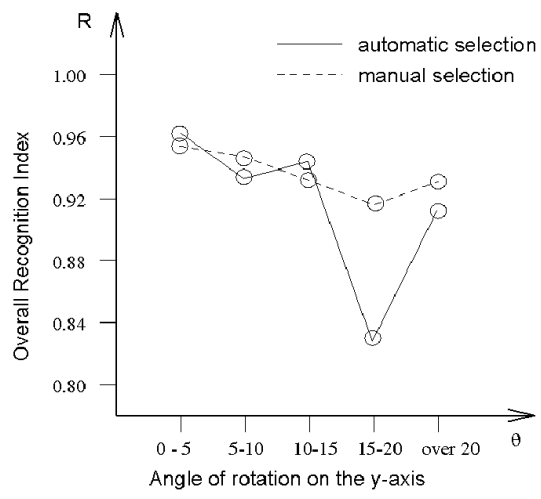

(b)

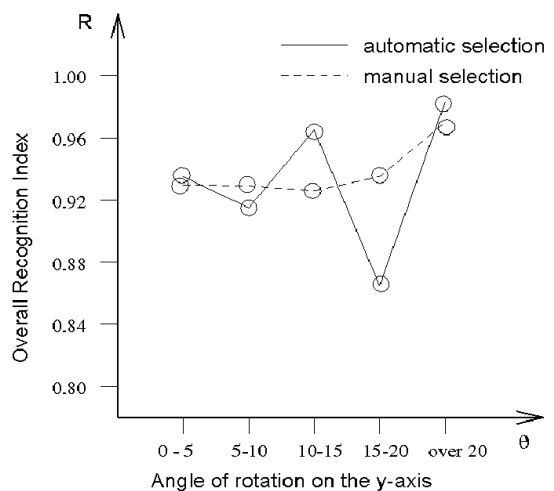

(c)

Fig. 16. The overall recognition index, $R$, at different angles of rotation, $\theta$, for (a) the eyes and eyebrows window, (b) the nose window, and (c) the mouth window.

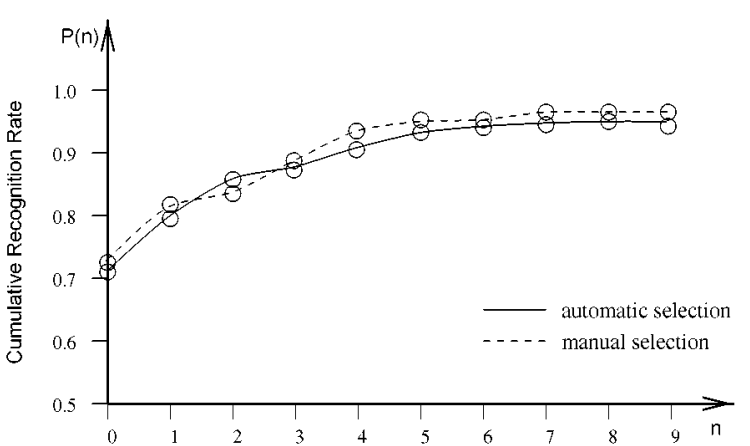

(a)

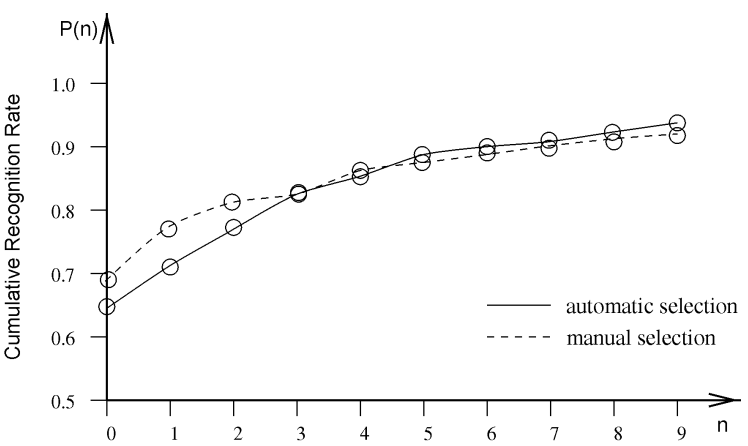

(b)

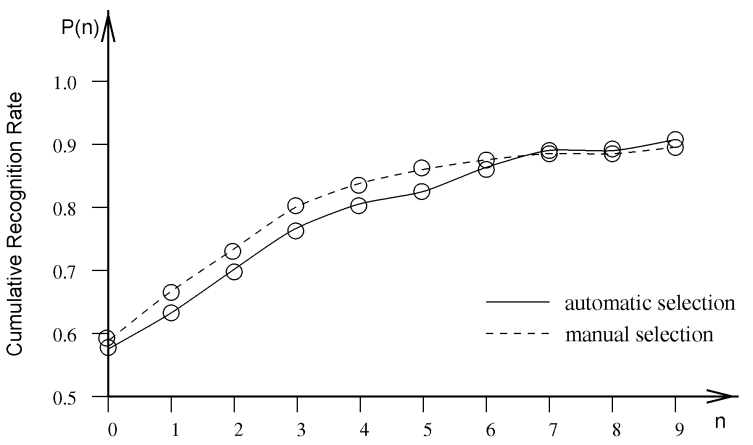

(c)

Fig. 17. The cumulative recognition rate, $P(n)$, for (a) the eyes and eyebrows window, (b) the nose window, and (c) the mouth window.

index, $R$, at different angles of rotation, $\theta$, and the cumulative recognition rate, $P(n)$, for automatic and manual feature selection procedures are illustrated in Figs. 16a, b, and c and Figs. 17a, b, and c, respectively. The respective overall recognition indices by considering all values of $\theta$ are tabulated in Table 3. From the results, the eyes and eyebrows window achieves the best performance which is less sensitive to the angle of rotation. The nose window shows a performance slightly inferior to that of the eyes and eyebrows window. This performance becomes worse when the angle of rotation increases. The mouth window exhibits the worst recognition performance. By separating the open- and closed-mouth, the closed-mouth images show a much better performance than the open-mouth images for recognition by correlation, as shown in Fig. 18 .

\subsection{Overall Performance}

The overall performance, by combining the point-matching method and correlation, is investigated. At the pointmatching stage, faces similar to the input are chosen from the database, and together with $D_{w}$, passed to the second stage which uses correlation for recognition. We assume that $N_{f}$ faces are selected at the first stage and their corresponding value of $D_{w}$ is denoted as $D_{w}(i)$, where $i=1, \cdots, N_{f}$. The minimum value among the $D_{w}(i)$ is denoted as $D_{w}^{\min }$.

At the second stage, the values of correlation for the three windows are computed and denoted as $g_{e}(i), g_{n}(i)$, and $g_{m}(i)$, respectively. The corresponding maximum values for these three terms are denoted as $g_{e}^{\max }, g_{n}^{\max }$, and $g_{m}^{\max }$, respectively. A cost function is set up to combine all the parts for recognition. 
TABLE 3

THE OVERALL RECOGNITION INDICES, R, OF FACIAL FEATURE WINDOWS

\begin{tabular}{|c|c|c|}
\hline \multirow{2}{*}{} & \multicolumn{2}{|c|}{ Overall recognition indices, $\mathrm{R}$} \\
\cline { 2 - 3 } & automatic & manual \\
\hline \hline eyes and eyebrows & 0.9571 & 0.9607 \\
\hline nose & 0.9426 & 0.9458 \\
\hline mouth & 0.9284 & 0.9317 \\
\hline
\end{tabular}

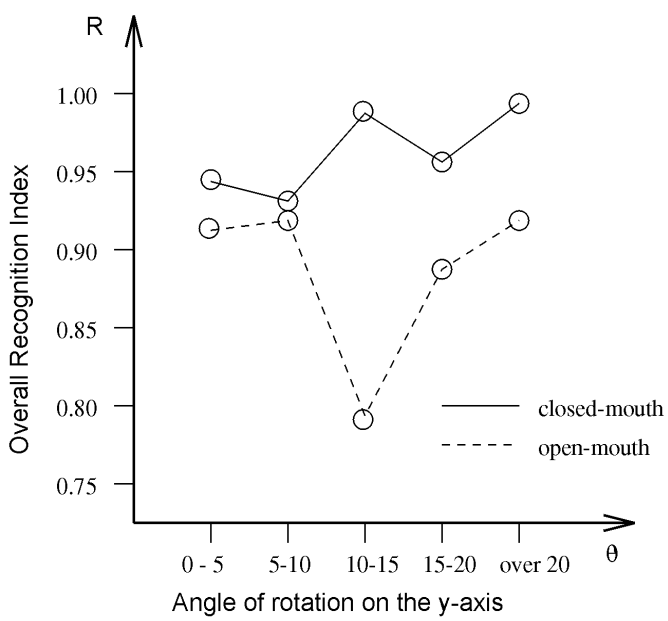

Fig. 18. The overall recognition index, $R$, of the closed-mouth, and the open-mouth images at different angle of rotation, $\theta$.

$$
C(i)=-k_{1} \cdot \frac{D_{w}^{\min }}{D_{w}(i)}-k_{2} \cdot \frac{g_{e}(i)}{g_{e}^{\max }}-k_{3} \cdot \frac{g_{n}(i)}{g_{n}^{\max }}-k_{4} \cdot \frac{g_{m}(i)}{g_{m}^{\max }}
$$

where $k_{1}, k_{2}, k_{3}$, and $k_{4}$ are weighting factors, and $i=1, \cdots, N_{f}$. Each of the terms is normalized to one so that the weighting factors can be set easily. In our experiments, $k_{1}=0.275$ and $k_{2}=0.30$, as these two terms exhibit a higher recognition rate than that of the nose and mouth parts. $k_{3}$ is set according to the angle of rotation. Because of the protrusion of the nose, geometric distortion results when it is rotated. Therefore, $k_{3}=0.290$ if the angle $\theta$ is less than $10^{\circ}$; otherwise, $k_{3}=$ 0.1 . This can reduce the effect of geometric distortion from the nose. The value of $k_{4}$ is set according to whether the mouth is open or closed. In the database, all the mouths are closed, so $k_{4}$ is set to a lower value if the mouth of the input face is detected to be open. For closed-mouth input, $k_{4}=$ 0.280 , and $k_{4}=0.075$ if the input has an open-mouth. Based on the value of the cost function, the one with minimum value is chosen as the best matched.

Fig. 19 shows the overall recognition indices, and the cumulative recognition rate at different angles of rotation, and the total cumulative recognition rate. As before, the performance of the overall system is insensitive to the angle of rotation, but outperforms the individual parts of the system. The variation in performance of the system using automatic and manual feature point selection procedures is slight.

The respective runtimes for these two recognition methods are also measured. The experiments were conducted on a Sun Sparcserver 1000. The point-matching stage exhibits a fairly constant speed, which is about 0.1 second on average.
For the correlation stage, the runtime depends on the number of faces passed to this stage. Fig. 20 shows the runtime of this stage in relation to the number of faces selected from the point-matching stage.

\section{Conclusions}

In this paper, we have adopted an analytic-to-holistic approach to face recognition. The positions of different facial feature points are located automatically or manually. This gives the information on where the facial features are and describes the overall configuration of a face. The feature point set of a test image is then compared to that in a database by a $2 \mathrm{D}$ point-matching scheme. Faces similar to the database are passed to the second stage, which matches faces by correlation. At this stage, the eyes and eyebrows window, nose window and mouth window are set up and compared to other faces by correlation. The correlation operation measures the similarity between the contents of the different windows. Experiments show that the overall performance by combining the two approaches is much better than either of them individually.

In order to cope with the effect of rotation of the face, a head model is introduced to reduce this effect on face recognition. By geometric measurements, we can estimate the angle of rotation of a face. Proper transformations and alignment can then be performed to obtain the approximate upright and frontal view of the faces. Experiments illustrate that this model can make both point-matching and correlation insensitive to the angle $\theta$.

For the 2D point-matching scheme, we have chosen 15 feature points, which can be detected automatically and reliably, and are significant for recognition. There are many other feature points which are also important for recognition. Some of them are: the two extreme points of each eyebrow, the two extreme points at the baseline of the nose, the position of the two ears, etc. However, these points are difficult to detect automatically and reliably. We believe that if these additional feature points can be included, the pointmatching scheme will perform much better.

In the database, each face is represented by 15 feature points, three facial feature windows, and the corresponding summation of gray-level intensity, $\Sigma_{i}\left|y_{i}\right|$, of each window for computing correlations. In conclusion, by combining the two different approaches, a much better recognition rate can be achieved. Using the automatic selection procedure, recognition rates of over 84 percent and 96 percent are obtained for considering the first and the first three likely matched faces, respectively. The corresponding recognition rates achieved using the manual selection procedure are over 89 percent and 97 percent, respectively. Thus, the difference in the performance of the system using automatic and manual feature point selection procedures is slight. The overall performance of the system also suggests that the different feature-based approaches and the holistic approaches may be combined, which will provide a much higher performance level than that of any individual approach. Furthermore, the total runtime for recognizing an input face is around 4-6 seconds on average; this includes the timings for facial feature extraction, face recognition by point-matching, and face recognition by correlation. 


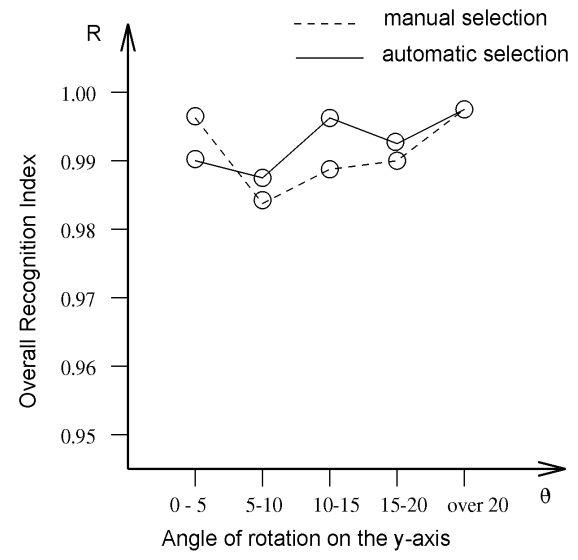

(a)

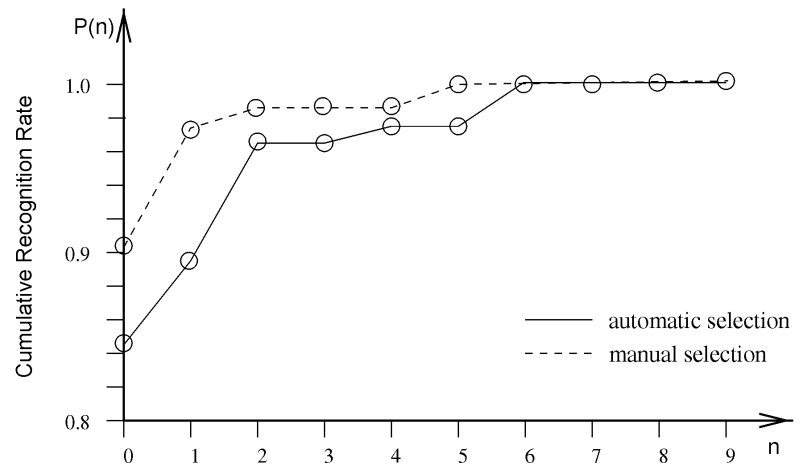

(b)

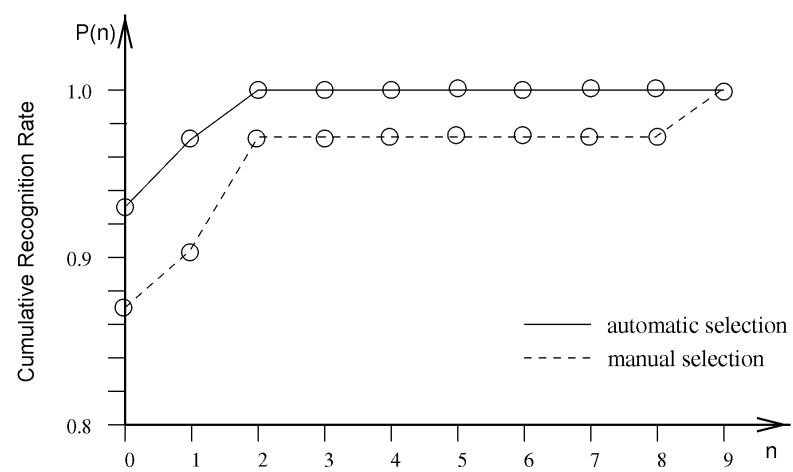

(d)

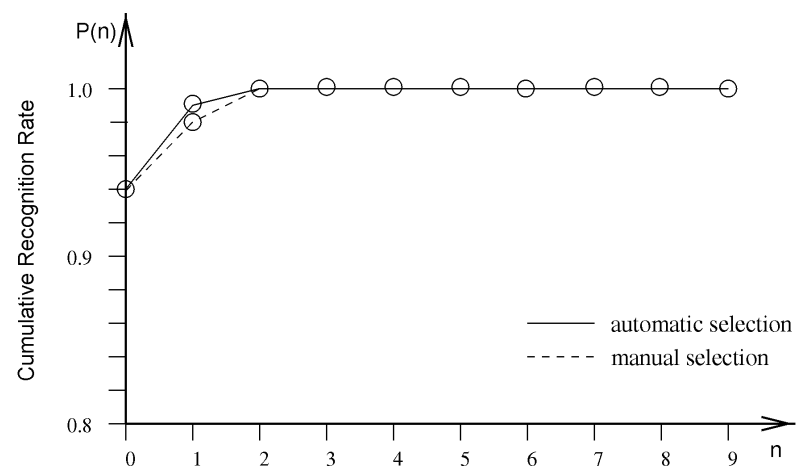

(f)

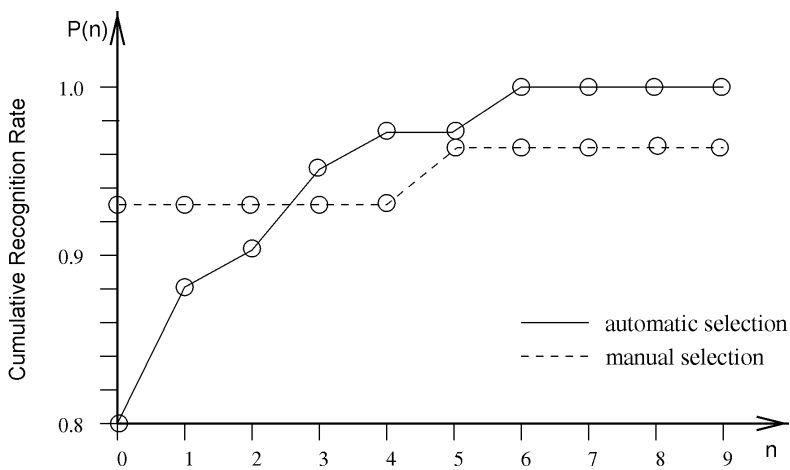

(c)

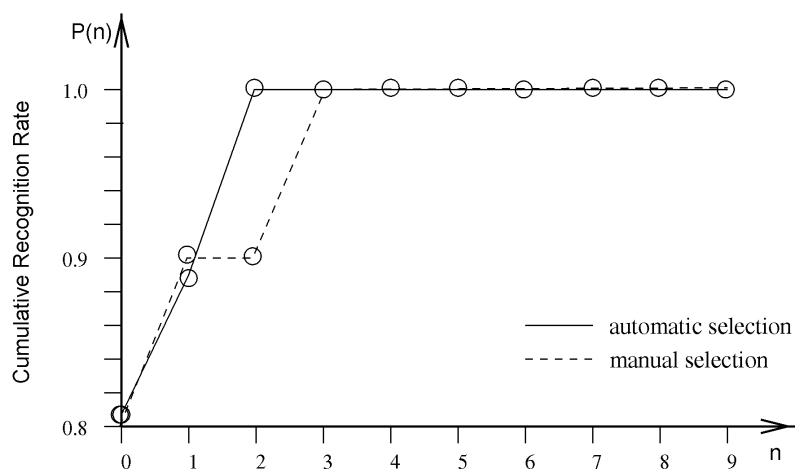

(e)

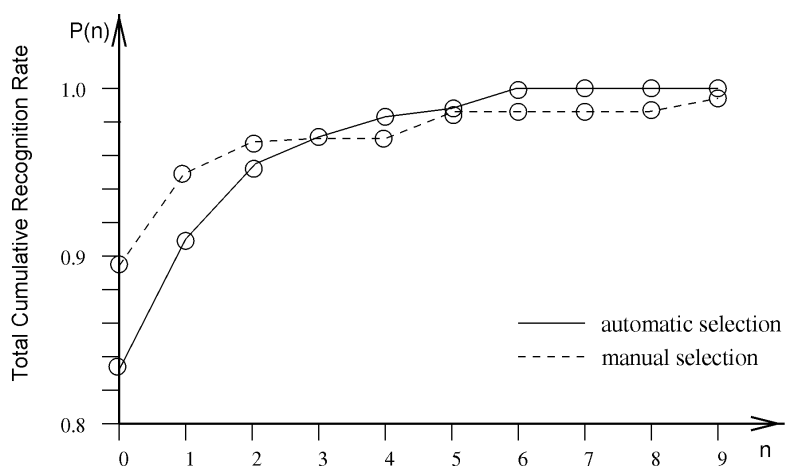

(g)

Fig. 19. (a) The overall recognition indices at different angles of rotation, and (b)-(f) the cumulative recognition rate at different angles of rotation and (g) the total cumulative recognition rate. (b) $0<\theta<5$. (c) $5<\theta<10$. (d) $10<\theta<15$. (e) $15<\theta<20$. (f) $\theta>20$. (g) All $\theta$. 


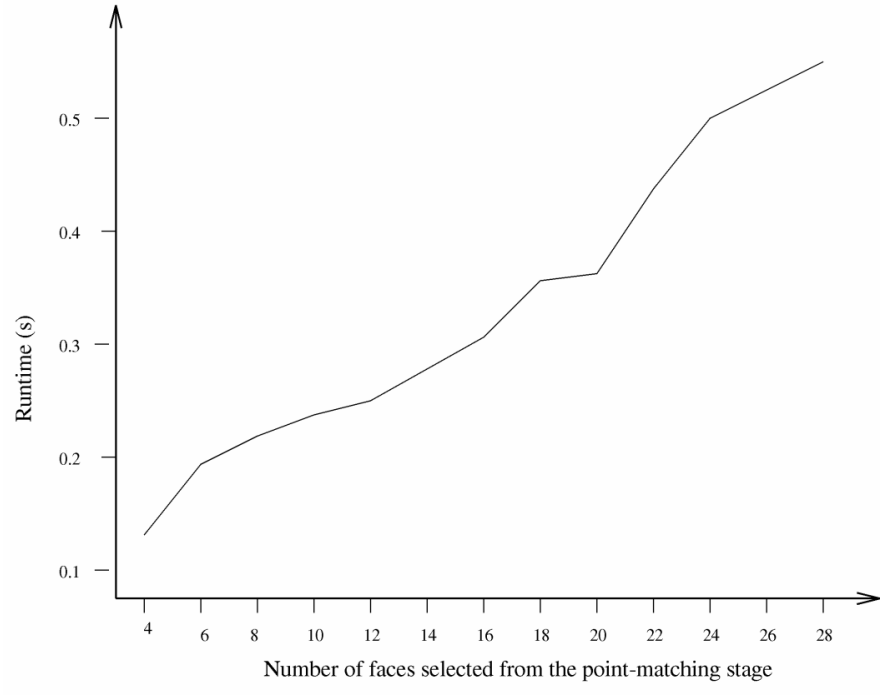

Fig. 20. Runtime of the correlation stage in relation to the number of faces selected from the point-matching stage.

\section{REFERENCES}

[1] A. Samal and P.A. Iyengar, "Automatic Recognition and Analysis of Human Faces and Facial Expressions: A Survey," Pattern Recognition, vol. 25, no. 1, pp. 65-77, 1992.

[2] M.A. Turk and A.P. Pentland, "Face Recognition Using Eigenfaces," Proc. CVPR, pp. 586-591, 1991.

[3] M.A. Turk and A.P. Pentland, "Eigenfaces for Recognition," J. Cognitive Neuroscience, vol.3, no.1, pp. 71-86, 1991.

[4] O. Nakamura, S. Mathur, and T. Minami, "Identification of Human Faces Based on Isodensity Maps," Pattern Recognition, vol. 24, no. 3, pp. 263-272, 1991.

[5] M. Lades, J.C. Vorbruggen, J. Buhmann, J. Lange, C.V.D. Malsburg, R.P. Wurtz, and W. Konen, "Distortion Invariant Object Recognition in the Dynamic Link Architecture," IEEE Trans. Computers, vol. 42, no. 3, pp. 300-311, Mar. 1993.

[6] J. Buhmann, J. Lange, and C.V.D. Malsburg, "Distortion Invariant Object Recognition by Matching Hierarchically Labeled Graphs," Proc. IJCNN'89, vol 1, pp. 155-159, 1989.

[7] Y.Q. Cheng, K. Liu, and J.Y. Yang, "A Novel Feature Extraction Method for Image Recognition Based on Similar Discriminant Function (SDF)," Pattern Recognition, vol. 26, no. 1, pp. 115-125, 1993.

[8] Z.Q. Hong, "Algebraic Feature Extraction of Image for Recognition," Pattern Recognition, vol. 24, no. 3, pp. 211-219, 1991.

[9] L.D. Harmon, S.C. Kuo, P.F. Ramig, and U. Raudkivi, "Identification of Human Face Profiles by Computer," Pattern Recognition, vol. 10, pp. 301-312, 1978.

[10] L.D. Harmon, M.K. Khan, R. Lasch, and P.F. Ramig, "Machine Identification of Human Faces," Pattern Recognition, vol. 13, no. 2, pp. 97-110, 1981.

[11] C.J. Wu and J.S. Huang, "Human Face Profile Recognition by Computer," Pattern Recognition, vol. 23, no. 3/4, pp. 255-259, 1990.

[12] C.W. Chen and C.L. Huang, "Human Face Recognition From a Single Front View," Int'l J. Pattern Recognition and Artificial Intelligence, vol. 6, no. 4, pp. 571-593, 1992.

[13] R. Brunelli and T.Poggio, "Face Recognition: Features Versus Templates," IEEE Trans. Pattern Analysis and Machine Intelligence, vol. 15, no. 10, pp. 1,042-1,052, 1993.

[14] M.S. Kamel, H.C. Shen, A.K.C. Wong, T.M. Hong, and R.I. Campeanu, "Face Recognition Using Perspective Invariant Features," Pattern Recognition Letters, no. 15, pp. 877-883, 1994.

[15] M. Kass, A. Witkin, and D. Terzopoulos, "Snakes: Active Contour Models," Int'l J. Computer Vision, vol. 1, pp. 321-331, 1987.

[16] K.M. Lam and H. Yan, "Fast Greedy Algorithm for Active Contours," Electronics Letters, vol. 30, no. 1, pp. 21-22, 1994.

[17] K.M. Lam and H. Yan, "Fast Algorithm for Locating Head Boundaries," J. Elec. Imaging, vol. 3, no. 4, pp. 351-359, 1994.
[18] X. Xie, R. Sudhakar, and H. Zhuang, "Corner Detection by a Cost Minimization Approach," Pattern Recognition, vol. 26, pp. 1,2351,243, 1993.

[19] K.M. Lam and H. Yan, "Facial Feature Location and Extraction for Computerized Human Face Recognition," Proc. Int'l Symp. Information Theory \& Its Applications, pp. 167-171, Nov. 1994.

[20] K.M. Lam and H. Yan, "Locating and Extracting the Eye in Human Face Images," Pattern Recognition, vol. 29, no. 5, pp. 771-779, 1996.

[21] A.L. Yuille, "Deformable Templates for Face Recognition," J. Cognitive Neuroscience, vol. 3, pp. 59-70, 1991.

[22] A.L. Yuille, P.W. Hallinan, and D.S. Cohen, "Feature Extraction From Faces Using Deformable Templates," Int'l J. Computer Vision, vol. 8, no. 2, pp. 99-111, 1992.

[23] K.M. Lam and H. Yan, "An Improved Method for Locating and Extracting the Eye in Human Face Images," ICPR'96, pp. C411C415, Aug. 1996.

[24] A.R. Mirhosseini, H. Yan, and K.M. Lam, "Adaptive Deformable Model for Mouth Boundary Detection," Optical Eng., vol. 37, no. 3, pp. 869-875, 1998.

[25] T.Kanade, "Picture Processing by Computer Complex and Recognition of Human Faces," tech. rep., Tyoto Univ., Dept. of Information Science, 1973.

[26] M. Werman and D. Weinshall, "Similarity and Affine Invariant Distances Between 2D Point Sets," IEEE Trans. Pattern Analysis and Machine Intelligence, vol. 17, no. 8, Aug. 1995.

[27] R. Brunelli and S. Messelodi, "Robust Estimation of Correlation With Applications to Computer Vision," Pattern Recognition, vol. 28, no. 6, pp. 833-841, 1995.

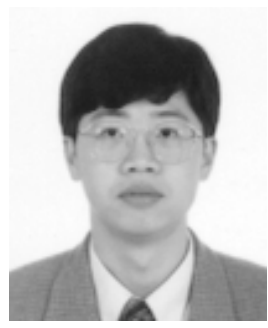

Kin-Man Lam received the associateship in electronic engineering from the Hong Kong Polytechnic University (formerly called Hong Kong Polytechnic) in 1986 and the MSc degree in communication engineering from the Department of Electrical Engineering, Imperial College of Science, Technology, and Medicine, England, in 1987. He joined TechTrend E. \& C. Ltd. as an application engineer working microsupercomputers and CAD/CAM. From 1990 to 1993, he was with the Department of Electronic Engineering at the Hong Kong Polytechnic University as a lecturer. In 1993, he undertook a PhD degree program in the Department of Electrical Engineering at the University of Sydney, Australia. He completed his PhD studies in August 1996. Since October 1996, he has been an assistant professor in the Department of Electronic Engineering at the Hong Kong Polytechnic University. His current research interests include image processing, computer vision and architecture, and pattern recognition.

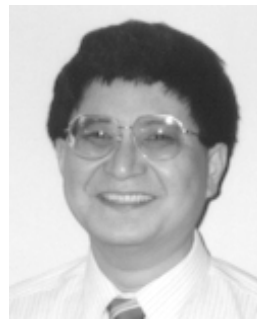

Hong Yan received his BE degree from Nanking Institute of Posts and Telecommunications in 1982, MSE degree from the University of Michigan in 1984, and PhD degree from Yale University in 1989, all in electrical engineering. From 1986 to 1989 , he was a research scientist at General Network Corporation, New Haven, Connecticut, where he worked on developing a CAD system for optimizing telecommunication systems. Since 1989, he has been with the University of Sydney, where he is currently a professor of electrical engineering. His research interests include medical imaging, signal and image processing, neural networks, and pattern recognition. $\mathrm{He}$ is an author or coauthor of one book and more than 150 technical papers in these areas. Dr. Yan is a fellow of the Institution of Engineers, Australia (IEAust), a senior member of the IEEE, and a member of the SPIE, the International Neural Network Society, the Pattern Recognition Society, and the International Society for Magnetic Resonance in Medicine. 